randomly selected from 9 regions in a population based casecontrol study in Spain (MCC-Spain study) whit demographic details, information on potential confounders and a comprehensive employment history. Each case of leukaemia may have one or more occupations. All occupation were codified by Occupational National Code (CNO 94) and The International Standard Classification of Occupations (ISCO-88) Associations between occupation and leukaemia were analysed using logistic regression adjusting for gender, age, and smoking.

Results We analysed the $27,4 \%$ of leukaemias.6\% never had occupation with risk of leukaemia and $41 \%$ were worked at least one occupation with probably exposition to carcinogens for leukaemia. Analysis is ongoing and results will be presented at the conference.

Conclusions In summary, our study showed some evidence supporting the role of some kind occupation in the development of leukaemia. However, given the relative low numbers the results have to be interpreted with some caution. On have analyse the exposition on these occupations.

\section{PRIMARY SELECTION AND IMPACT OF SHIFT WORK ON CARDIOVASCULAR RISK FACTORS}

Mei Yong, Michael Nasterlack, Christina Germann, Rolf-Peter Pluto, Stefan Lang, Christoph Oberlinner. Department of Occupational Medicine and Health Protection, BASF SE, Ludwigshafen, Germany

\subsection{6/oemed-2014-102362.371}

Objectives A potential "healthy worker effect" may bias the studied effect of shift work on health. The observed differ-ences in health behaviour and health outcomes might be caused by: (i) primary selection, (ii) influence from the with shift work related environment, and (iii) impact of shift work. We aimed to study these potential sources.

Method A cohort of 4754 male trainees who had finished their professional training and started their career in the production between 1995 and 2012 was identified. Among them, 1348 (28\%) were involved in rotating shift work and 3406 (72\%) in a day working time. Information on lifestyle and blood pressure (BP), body mass index, and measurements of laboratory parameters were retrieved from the data of medical examinations. These were then compared at (i) the beginning, (ii) the end of training, and (iii) 3 years after the job begin, in relation to the working time.

Results At the beginning of the training, the prevalence of smokers was higher among shift workers (26\% vs. $21 \%)$. During the training and the first 3 years of job, marginal decline of systolic BP and elevation of triglyceride were observed among the shift workers, after the adjustment for age at begin, duration between the exami-nations, and the measurements at the baseline. No difference was found with respect to other risk factors for cardiovascular diseases.

Conclusions Our findings do not support a primary selection in favour of shift workers. A potential impact of shift work on lifestyle and health will be studied prospectively.

\section{DIFFERENCES IN OCCUPATIONAL INJURY RATES BY AGE GROUP, INDUSTRY AND INJURY TYPE}

${ }^{1,2}$ Kenneth Scott. 'Colorado School of Public Health, Department of Epidemiology, Aurora, CO, USA; ${ }^{2}$ Mountain and Plains Education and Research Center, Aurora, CO, USA

10.1136/oemed-2014-102362.372
Objectives The US workforce, like workforces around the globe, is ageing - which creates new challenges for occupational health researchers and practitioners. Throughout their working lives, workers experience changes in occupational exposures, behaviours and time demands. The ways in which age-related changes in specific injury risk factors interact to influence injury risk are complex. Data reported in Morbidity and Mortality Weekly Reports Data collected by the United States Bureau of Labour Statistics was analysed to compare age-related differences in occupational injury rates across several industrial sectors and subsectors.

Method Occupational injury incidence rate estimates were developed to compare age-related patterns by industry and injury type. Injury count data from 2010, stratified by age group, industry sector and injury type, were provided by the US Bureau of Labour Statistics Survey of Occupational Injuries and Illnesses. The Current Population Survey was used to develop estimates of at-risk experience. Data from a diverse collection of industry subgroups (defined by NAICS codes) were analysed, including agriculture, transportation and warehousing, private hospitals, nursing and residential care facilities, police protection and construction.

Results There are significant differences in the rates of occupational injuries when stratified by age group, industry and injury type.

Conclusions Additional research should explore the reasons why occupational injury rates differ by age group, industry and injury type. Occupational safety programs and policies should consider age-related differences in injury risks when allocating resources toward prevention efforts.

\section{A SYSTEMIC REVIEW AND META-ANALYSIS OF EXHALED NITRIC OXIDE IN CHRONIC OBSTRUCTIVE PULMONARY DISEASE: RELATIONSHIP TO PULMONARY FUNCTION}

${ }^{1}$ Se Yeong Kim, ${ }^{1}$ Shin Ae KIM, ${ }^{1}$ Chunhui Suh, ${ }^{1}$ Kun Hyung Kim, ${ }^{1}$ Chae Kwan Lee, ${ }^{1}$ Byung Chul Son, ${ }^{1}$ Jeong Ho Kim, ${ }^{1}$ Jong Tae Lee, ${ }^{2}$ Jung IL Kim. ${ }^{1}$ Department of Occupational and Environmental Medicine and Institute of Environmental and Occupational Medicine, Busan Paik Hospital, Inje University, Busan/Busanjin-Gu, Republic of Korea; ${ }^{2}$ Department of Occupational and Environmental Medicine and Institute of Environmental and Occupational Medicine, Busan Paik Hospital, Dong-a University, Busan/Seo-Gu, Republic of Korea

\subsection{6/oemed-2014-102362.373}

Objectives Fractional exhaled nitric oxide (FeNO) has been implicated as a pulmonary biomarker in various respiratory disease, including COPD. Measurement of FeNO is a simple, noninvasive tool for assessing airway inflammation. Neverthless, the usefulness of FeNO measurements in COPD patient in clinical practice is unclear. The objective of this review was to evaluate the efficacy of management of COPD based on FeNO in comparison with pulmonary function test.

Method Cochrane library (CENTRAL), MEDLINE, EMBASE and reference lists of articles were searched. The last searches were in July 2013. Results of searches were reviewed against predominantly criteria for inclusion. Relevant studies were selected, assessed and data extracted independently by two people. Participant articles with COPD management based on pulmonary function test compared with $\mathrm{FeNO}$ measurement were selected. Risk of bias for each study was assessed using the QUADAS(quality assessment of studies of diagnosis accuracy included in systematic reviews) scale.

Results Finally, eight studies were included. Of the eight studies, four were a negative and one were positive correlation between 